Nuclear Physics A442 (1985) 50-67

(C) North-Holland Publishing Company

\title{
COLLECTIVE PAIRING HAMILTONIAN IN THE GCM APPROXIMATION ${ }^{\dagger}$
}

\author{
A. GÓŹDŹ*, K. POMORSKI*, M. BRACK and E. WERNER \\ Institute of Theoretical Physics, University of Regensburg, D-8400 Regensburg, West Germany \\ Received 29 January 1985 \\ (Revised 25 March 1985)
}

\begin{abstract}
Using the generator coordinate method and the gaussian overlap approximation we derived the collective Schrödinger-type equation starting from a microscopic single-particle plus pairing hamiltonian for one kind of particle. The BCS wave function was used as the generator function. The pairing energy-gap parameter $\Delta$ and the gauge transformation angle $\phi$ were taken as the generator coordinates. Numerical results have been obtained for the full and the mean-field pairing hamiltonians and compared with the cranking estimates. A significant role played by the zero-point energy correction in the collective pairing potential is found. The ground-state energy dependence on the pairing strength agrees very well with the exact solution of the Richardson model for a set of equidistant doubly-degenerate single-particle levels.
\end{abstract}

\section{Introduction}

The concept of a collective pairing hamiltonian was introduced by Bès et al. ${ }^{1}$ ). These authors used as collective variables the intrinsic pairing deformation $\alpha$, related to the gap parameter $\Delta$, and the gauge angle $\phi$. A collective hamiltonian was then derived from the mean-field pairing hamiltonian within the cranking approximation. The collective parameter $\alpha$ (or $\Delta$ ) was responsible for the occurrence of pairing vibrations, and with the gauge angle $\phi$ quasirotational bands were connected.

The aim of the present paper is to derive a collective pairing hamiltonian using the generator coordinate method (GCM) in the generalized gaussian overlap approximation $(\mathrm{GOA})^{2,3}$ ). The GCM derivation of the collective hamiltonian was already discussed in ref. ${ }^{3}$ ) for a system described by a mean-field hamiltonian. Using the simplest possible two-body hamiltonian, we shall now test the mean pairing field approach and the quality of the BCS functions as generator functions. It is not our aim here to reproduce experimental data, but to treat the pairing problem as an example for testing our approach. Collective pairing vibrations were already in-

† Work supported in parts by GSI Darmstadt and Deutsche Forschungsgemeinschaft Bonn.

* On leave of absence from: Department of Theoretical Physics, University MCS, Lublin, Poland. 
vestigated within the GCM [see e.g. refs. ${ }^{4,5}$ )] but the Hill-Wheeler equation was solved numerically and no collective hamiltonian was derived. The pairing energy gap $\Delta$ [and $\lambda$ in ref. ${ }^{4}$ )] was the generating variable and the particle-number-projected BCS wave function was used as the generator function there.

In contrast to that approach, we use here two generator coordinates, as in refs. ${ }^{1,6}$ ), namely the gap parameter $\Delta$ and the gauge angle $\phi$ which is the canonically conjugate variable to the particle-number operator. Our slightly modified BCS function is [see also ref. $^{1}$ )]

$$
|\Delta \phi\rangle=\mathrm{e}^{i N \phi} \prod_{\nu>0}\left[u_{\nu}(\Delta)+\mathrm{e}^{-2 i \phi} v_{\nu}(\Delta) c_{\nu}^{+} c_{-\nu}^{+}\right]|0\rangle,
$$

where $N$ is the average number of particles

$$
N=\langle\Delta \phi|\hat{N}| \Delta \phi\rangle .
$$

The use of the gauge angle $\phi$ as a generator coordinate corresponds to an approximate particle-number projection.

For the formal derivation of the GOA, it is necessary ${ }^{8}$ ) to start with "cartesian" coordinates $\Delta_{x}, \Delta_{y} \in(-\infty,+\infty)$ as collective variables instead of the "polar" variables $(\Delta, 2 \phi)$ with $\Delta \in(0, \infty), \phi \in[0, \pi]$. The results are, however, always expressed in terms of $\Delta$ and $\phi$.

The overlap of two functions (1.1) is complex and can be written in the following form:

$$
\begin{aligned}
\left\langle\Delta \phi \mid \Delta^{\prime} \phi^{\prime}\right\rangle= & \exp \left[i \Phi\left(\Delta, \phi, \Delta^{\prime}, \phi^{\prime}\right)\right] \\
& \times \prod_{\nu>0}\left[u_{\nu}^{2} u_{\nu}^{\prime 2}+v_{\nu}^{2} v_{\nu}^{\prime 2}+2 u_{\nu} v_{\nu} u_{\nu}^{\prime} v_{\nu}^{\prime} \cos 2\left(\phi-\phi^{\prime}\right)\right]^{1 / 2},
\end{aligned}
$$

where

$$
\Phi\left(\Delta, \phi, \Delta^{\prime}, \phi^{\prime}\right)=N\left(\phi^{\prime}-\phi\right)-\sum_{\nu>0} \operatorname{arctg}\left[\frac{v_{\nu} v_{\nu}^{\prime} \sin 2\left(\phi-\phi^{\prime}\right)}{u_{\nu} u_{\nu}^{\prime}+u_{\nu} u_{\nu}^{\prime} \cos 2\left(\phi-\phi^{\prime}\right)}\right] .
$$

(The prime above the $u$ - and $v$-coefficients denotes here dependence on $\Delta^{\prime}$.) Of course, in the case of complex overlap functions, the derivation of the collective hamiltonian described in refs. ${ }^{2,3}$ ) has to be generalized; this is shown in sect. 3 .

The microscopic hamiltonian and the microscopic formulae for the parameters of the collective hamiltonian are presented in appendix A. In sect. 3 the collective potential and the mass parameters obtained with the full and the mean-field pairing hamiltonian are compared with the cranking estimates. We also compare our approximate eigenstates to the exact solution of the pairing problem for a set of 32 equidistant levels with 32 particles $^{9}$ ). The numerical method of diagonalizing the collective pairing collective hamiltonian is described in appendix B. 


\section{The extended gaussian overlap approximation for complex overlap functions}

Let us consider the following model overlap function:

$$
\left\langle a \mid a^{\prime}\right\rangle \approx \mathcal{N}\left(a, a^{\prime}\right)=\mathrm{e}^{i \Phi\left(a, a^{\prime}\right)} \exp \left\{-\frac{1}{2} \sum_{\kappa=1}^{n}\left(\Gamma^{\kappa}\left(a, a^{\prime}\right)\right)^{2}\right\},
$$

where $a\left(a^{\prime}\right)$ denotes a set of $n$ collective variables (in the pairing case $a=\{\Delta, \phi\}$ and $\left.a^{\prime}=\left\{\Delta^{\prime}, \phi^{\prime}\right\}\right)$. Following refs. ${ }^{2,3}$ ), the metric tensor in the collective space is defined as

$$
\gamma_{\mu \nu}(q)=-\left(\frac{\partial}{\partial s^{\mu}} \frac{\partial}{\partial s^{\nu}} \mathcal{N}\left(q+\frac{1}{2} s, q-\frac{1}{2} s\right)\right)_{s=0},
$$

where $q=\frac{1}{2}\left(a+a^{\prime}\right)$ and $s=a-a^{\prime}$. The metric tensor will be real if we assume that

$$
\Phi(q, q)=\left(\frac{\partial \Phi\left(q+\frac{1}{2} s, q-\frac{1}{2} s\right)}{\partial s^{\mu}}\right)_{s=0}=\left(\frac{\partial^{2} \Phi\left(q+\frac{1}{2}, q-\frac{1}{2} s\right)}{\partial s^{\mu} \partial s^{\nu}}\right)_{s=0}=0,
$$

where $q^{1}=\frac{1}{2}\left(\Delta+\Delta^{\prime}\right), q^{2}=\frac{1}{2}\left(\phi+\phi^{\prime}\right), s^{1}=\Delta-\Delta^{\prime}$, and $s^{2}=\phi-\phi^{\prime}$.

A straightforward calculation shows that eq. (2.3) is true for the generating function (1.1) and the phase (1.4). So in the following we assume the same property of the exponent $\phi\left(a, a^{\prime}\right)$ in the more general case considered here. Eqs. (2.2) and (2.3) lead then to the same expression for the metric tensor as in the case of a real overlap function $\left.{ }^{2,3}\right)$ :

$$
\gamma_{\mu \nu}(a)=\sum_{\kappa=1}^{n} \frac{\partial \Gamma^{\kappa}\left(a, a_{0}\right)}{\partial a^{\mu}} \frac{\partial \Gamma^{\kappa}\left(a, a_{0}\right)}{\partial a^{r}},
$$

where $a_{0}$ is a fixed point in the collective space.

To obtain the collective hamiltonian $\hat{\mathscr{H}}_{\text {coll }}$ we consider the expectation value of the many-body hamiltonian $\hat{H}$ between the GCM wave functions defined as usual in the form

$$
|\Psi\rangle=\int \mathrm{d} a f(a)|a\rangle
$$

In the case of pairing forces the generating function is $|a\rangle=|\Delta \phi\rangle$, eq. (1.1). The expectation value of $\hat{H}$ can be written now as a doubly-multidimensional integral:

$$
\langle\Psi|\hat{H}| \Psi\rangle=\int \mathrm{d} a \mathrm{~d} a^{\prime} f^{*}(a) h\left(a, a^{\prime}\right) f\left(a^{\prime}\right) \mathscr{N}\left(a, a^{\prime}\right),
$$

where the reduced energy overlap is defined by the equation

$$
h\left(a, a^{\prime}\right)=\frac{\langle a|\hat{H}| a\rangle}{\left|\mathcal{N}\left(a, a^{\prime}\right)\right|} .
$$

Note the difference between the standard definition of $h\left(a, a^{\prime}\right)\left[\right.$ ref. $\left.\left.{ }^{8}\right)\right]$ and eq. (2.7). 
We need here only the absolute value of $\mathscr{N}\left(a, a^{\prime}\right)$ and not $\mathscr{N}\left(a, a^{\prime}\right)$ itself. Because the hamiltonian is hermitian, $h\left(a, a^{\prime}\right)$ eq. (2.7) is also hermitian, i.e. $h\left(a, a^{\prime}\right)^{*}=$ $h\left(a^{\prime}, a\right)$.

The next step is a change of variables in the integral (2.6) leading to the gaussian form of the overlap function $\left|\mathscr{N}\left(a, a^{\prime}\right)\right|$. The appropriate transformation is given by $^{2,3}$ )

$$
\alpha^{\kappa}=\Gamma^{\kappa}\left(a, a_{0}\right), \quad \kappa=1,2, \ldots, n .
$$

We perform a Taylor expansion of the reduced energy overlap in the variables $\left\{\alpha^{\kappa}\right\}$ around an arbitrary point $\xi$ up to second order. Following the method described in detail in subsect. 3.2 of $^{\text {ref. }}{ }^{3}$ ), we finally obtain the collective hamiltonian. After a transformation back to the original collective variables, we obtain

$$
\hat{\mathscr{H}}_{\text {coll }}=\hat{T}+\hat{F}+\hat{V} \text {. }
$$

Here $\hat{T}$ and $\hat{V}$ correspond to the collective kinetic and potential energies respectively. The meaning of the additional term $\hat{F}$, absent in the case of the collective cranking hamiltonian, will be explained below. In the covariant notation the kinetic energy term is given by

$$
\hat{T}=-\frac{1}{2} \frac{1}{\sqrt{\gamma}} \frac{\partial}{\partial q^{\nu}}\left(\mathscr{M}^{-1}(q)\right)^{\nu \nu^{\prime}} \frac{\partial}{\partial q^{\nu^{\prime}}}
$$

with the inverse mass tensor

$$
\left(\mathscr{M}^{-1}(q)\right)_{\mu \nu}=-\frac{1}{2}\left[\operatorname{Re}\left(\frac{\Delta}{\Delta a^{\mu}} \frac{\Delta h\left(a, a^{\prime}\right)}{\Delta a^{\nu}}\right)-\frac{\Delta}{\Delta a^{\mu}} \frac{\Delta h\left(a, a^{\prime}\right)}{\Delta a^{\prime \nu}}\right]_{a=a^{\prime}=q} .
$$

The potential energy is

$$
V=\langle q|\hat{H}| q\rangle-\varepsilon_{0}(q)
$$

and the so-called zero-point energy $\varepsilon_{0}(q)$ can be expressed as

$$
\varepsilon_{0}(q)=\frac{1}{2} \gamma^{\mu \nu} \operatorname{Re}\left[\frac{\Delta}{\Delta q^{\nu}}\left(\frac{\Delta h\left(a, a^{\prime}\right)}{\Delta a^{\mu}}\right)_{q}-\left(\frac{\Delta}{\Delta a^{\mu}} \frac{\Delta h\left(a, a^{\prime}\right)}{\Delta a^{\nu}}\right)_{q}\right] .
$$

The hermitian operator $\hat{F}$ has the following form,

$$
\hat{F}=-\frac{1}{2} i \gamma^{\mu \nu} \operatorname{Im}\left[\frac{\Delta}{\Delta q^{\nu}}\left(\frac{\Delta h\left(a, a^{\prime}\right)}{\Delta a^{\mu}}\right)_{q}\right]-i \gamma^{\mu \nu} \operatorname{Im}\left(\frac{\partial h\left(a, a^{\prime}\right)}{\partial a^{\mu}}\right)_{q} \frac{\partial}{\partial q^{\nu}},
$$

and its systematical investigation should allow one to classify new collective effects which are not described in the framework of the cranking model with Pauli quantization ${ }^{10}$ ).

In the case of the pairing hamiltonian, the term $\hat{F}$, which is linear in the derivatives with respect to the collective variables, is related to the experimentally observed asymmetry between the pairing rotational bands for nuclei with $N, N+$ $2, N+4, \ldots$ and $N, N-2, N-4, \ldots$.

In the eqs. (2.11)-(2.14), $\Delta / \Delta q^{\mu}$ denotes the covariant derivative and $\gamma^{\mu \nu}$ stands for the contravariant components of the metric tensor. 


\section{Results and discussion}

\subsection{COLLECTIVE PAIRING HAMILTONIAN}

The collective hamiltonian derived above in eqs. (2.9)-(2.14) can be rewritten in the variables $\Delta$ and $\phi$ :

$$
\begin{aligned}
\hat{\mathscr{H}}_{\text {coll }}= & -\frac{\hbar^{2}}{2 \sqrt{\operatorname{det} \gamma}} \frac{\partial}{\partial \Delta} \sqrt{\operatorname{det} \gamma} \mathscr{M}_{\Delta \Delta}^{-1} \frac{\partial}{\partial \Delta}-\frac{1}{2} \hbar^{2} \mathscr{M}_{\phi \phi}^{-1} \frac{\partial^{2}}{\partial \phi^{2}} \\
& -i \hbar \frac{\operatorname{Im}\left\langle\Delta \phi\left|\frac{\grave{\partial}}{\partial \phi} \hat{H}\right| \Delta \phi\right\rangle}{\gamma_{\phi \phi}} \frac{\partial}{\partial \phi}+V(\Delta) .
\end{aligned}
$$

The first term describes the kinetic vibrational energy, the second term gives the so-called pairing rotational band, a multiplet of states with the particle number $N, N+2, N \pm 4 \ldots$, and the third term gives the asymmetry of these bands. In deriving eq. (3.1) from (2.9)-(2.14), we have used the fact that all microscopic coefficients depend on $\Delta$ only. The widths of the gaussian overlap are

$$
\begin{array}{ll}
\gamma_{\Delta \Delta}=\left\langle\Delta \phi\left|\frac{\grave{\partial}}{\partial \Delta} \frac{\vec{\partial}}{\partial \Delta}\right| \Delta \phi\right\rangle, & \gamma_{\Delta \phi}=0, \\
\gamma_{\phi \phi} & =\left\langle\Delta \phi\left|\frac{\grave{\partial}}{\partial \phi} \frac{\vec{\partial}}{\partial \phi}\right| \Delta \phi\right\rangle .
\end{array}
$$

The inverse mass parameter tensor is also diagonal with

$$
\begin{aligned}
\mathscr{M}_{\Delta \Delta}^{-1}= & \left\{\left\langle\Delta \phi\left|\frac{\grave{\partial}}{\partial \phi} \hat{H} \frac{\vec{\partial}}{\partial \phi}\right| \Delta \phi\right\rangle_{\mathrm{L}}-\frac{1}{2} \frac{\partial}{\partial \Delta}\left\langle\Delta \phi\left|\hat{H} \frac{\vec{\partial}}{\partial \Delta}\right| \Delta \phi\right\rangle\right. \\
& \left.+\frac{1}{4} \frac{\partial \ln \gamma_{\Delta \Delta}}{\partial \Delta}\left\langle\Delta \phi\left|\hat{H} \frac{\vec{\partial}}{\partial \Delta}\right| \Delta \phi\right\rangle\right\} / \gamma_{\Delta \Delta}^{2}, \\
\mathscr{M}_{\phi \phi}^{-1}= & \left\{\left\langle\Delta \phi\left|\frac{\grave{\partial}}{\partial \phi} \hat{H} \frac{\vec{\partial}}{\partial \phi}\right| \Delta \phi\right\rangle_{\mathrm{L}}-\frac{1}{4 \gamma_{\Delta \Delta}} \frac{\partial \gamma_{\phi \phi}}{\partial \Delta}\left\langle\Delta \phi\left|\hat{H} \frac{\vec{\partial}}{\partial \Delta}\right| \Delta \phi\right\rangle\right\} / \gamma_{\phi \phi}^{2} .
\end{aligned}
$$

The so-called linked matrix element is defined as ${ }^{8}$ )

$$
\left\langle q\left|\frac{\grave{\partial}}{\partial q} \hat{H} \frac{\vec{\partial}}{\partial q}\right| q\right\rangle_{\mathrm{L}} \equiv\left\langle q\left|\frac{\grave{\partial}}{\partial q} \hat{H} \frac{\vec{\partial}}{\partial q}\right| q\right\rangle-\left\langle q\left|\frac{\grave{\partial}}{\partial q} \frac{\vec{\partial}}{\partial q}\right| q\right\rangle\left\langle q \mid \hat{H}_{q}\right\rangle,
$$

where the arrows denote on which side the operator $\partial / \partial q$ acts. The collective pairing potential is equal to

$$
V=\langle\Delta \phi|\hat{H}| \Delta \phi\rangle-\varepsilon_{0}
$$


where the zero-point energy is given by

$$
\begin{aligned}
\varepsilon_{0}= & \frac{1}{2}\left\{\left(\left\langle\Delta \phi\left|\frac{\grave{\partial}}{\partial \Delta} \hat{H} \frac{\vec{\partial}}{\partial \Delta}\right| \Delta \phi\right\rangle_{\mathrm{L}}+\left\langle\Delta \phi\left|\frac{\partial \hat{H}}{\partial \Delta} \frac{\vec{\partial}}{\partial \Delta}\right| \Delta \phi\right\rangle\right) / \gamma_{\Delta \Delta}\right. \\
& \left.+\left(\left\langle\Delta \phi\left|\frac{\grave{\partial}}{\partial \phi} \hat{H} \frac{\vec{\partial}}{\partial \phi}\right| \Delta \phi\right\rangle_{\mathrm{L}}+\left\langle\Delta \phi\left|\frac{\partial \hat{H}}{\partial \phi} \frac{\vec{\partial}}{\partial \phi}\right| \Delta \phi\right\rangle\right) / \gamma_{\phi \phi}\right\} .
\end{aligned}
$$

The terms containing $\partial \hat{H} / \partial \Delta$ and $\partial \hat{H} / \partial \phi$ are nonzero only for a mean-field hamiltonian which depends explicitly on the collective variables ${ }^{3}$ ).

Our microscopic calculations were performed (i) for a full pairing hamiltonian

$$
\hat{H}=\hat{H}_{0}-G \hat{P}^{+} \hat{P},
$$

where $\hat{H}_{0}$ is the single-particle hamiltonian and

$$
\hat{P}=\sum_{\nu>0} c_{-\nu} c_{\nu}
$$

and (ii) for a mean-field pairing hamiltonian

$$
\hat{H}_{\mathrm{MF}}=\hat{H}_{0}-G\left(\left\langle\hat{P}^{+}\right\rangle \hat{P}+\hat{P}^{+}\langle\hat{P}\rangle\right)+G\left\langle\hat{P}^{+}\right\rangle\langle\hat{P}\rangle .
$$

The average \langle\rangle in (3.11) is taken between the BCS wave functions (1.1). The detailed microscopic expressions for the quantities (3.2)-(3.8) are derived in appen$\operatorname{dix} \mathbf{A}$ for both cases.

\subsection{NUMERICAL RESULTS IN AN EXACTLY SOLUBLE MODEL}

We have used the Richardson model of equidistant doubly-degenerate levels in order to test our collective model. As an example was used 32 levels filled by 32 particles. The (dimensionless) energies of the levels are $e_{i}=i$, where $i=1,2 \cdots 32$. In ref. ${ }^{9}$ ) the exact solutions for the hamiltonian $\hat{H},(3.9)$, are given for this case. We compare these solutions for the different pairing strengths $G$ with the results of our model.

The GCM potential energy (3.7) corresponding to the full pairing hamiltonian (3.9), as well as the mean-field hamiltonian (3.11), and the BCS ground-state energy [i.e. the potential in the cranking case $\left.^{1}\right)$ ] are plotted in fig. 1 . Due to the zero-point energy correction the GCM potential is much more deformed than the BCS potential. Note that also for the mean-filled hamiltonian, $\varepsilon_{0}$ is not equal to zero here as it was the case for the Hartree-Fock-type hamiltonians in ref. $^{3}$ ). This is so because the hamiltonian (3.11) is not the free quasiparticle hamiltonian.

The value of the pairing gap $\Delta$ at equilibrium is almost two times larger for the GCM potential than for the BCS case; also the deformation energy is almost 7 times larger for the GCM potential. However, a first glance at the mass parameters $\left(\mathscr{M}_{\Delta \Delta}\right)$ and their dependence on $\Delta$, shown in fig. 2 , is sufficient to convince us that the 


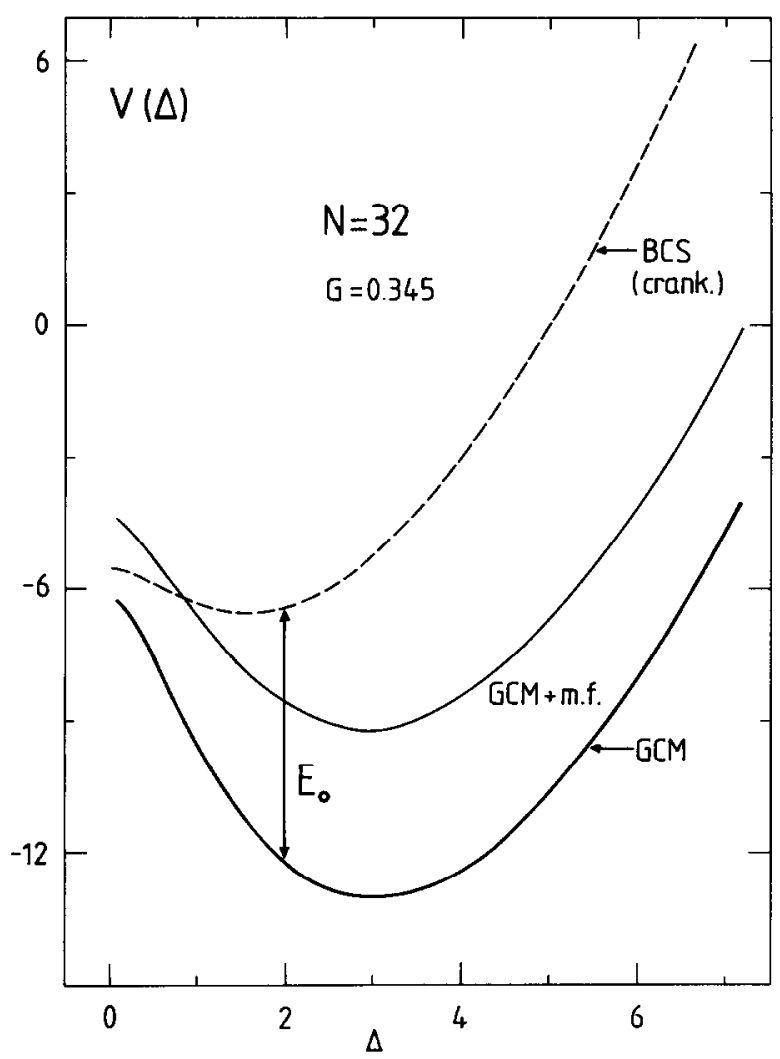

Fig. 1. Potential cncrgy for the Richardson model as a function of $\Delta$. The thick solid line corresponds to the GCM potential obtained for the full pairing hamiltonian, and the thin line represents the result for the mean-field hamiltonian. The dashed curve denotes the BCS energy which plays the role of the potential in the cranking hamiltonian. $V$ and $\Delta$ are in units of the level spacing.

motion in the collective potential energy well is strongly anharmonic and our usual experience fails. The mass parameters $\mathscr{M}_{\Delta \Delta}$ are essentially inversely proportional to $\Delta^{2}$ (see eq. (89)).

At the BCS equilibrium point, both in the GCM and in the mean-field approach, $\mathscr{M}_{\Delta \Delta}$ is around $\frac{1}{2}$ of the cranking estimate while the mass for the full pairing hamiltonian is already $\frac{2}{3}$ of the cranking mass.

The second component of the mass tensor $\mathscr{M}_{\phi \phi}$, the "quasi moment of inertia", does not vary with $\Delta$ as strongly as $\mathscr{M}_{\Delta \Delta}$; in the cranking case it has even a broad plateau, as seen in fig. 3 . This dependence of the cranking masses $\mathscr{M}_{\Delta \Delta}$ and $\mathscr{M}_{\phi \phi}$ contradicts the assumptions made in ref. $^{1}$ ).

The widths $\gamma_{\Delta \Delta}$ and $\gamma_{\phi \phi}$ of the overlap of the BCS functions are plotled in fig. 4. The $\gamma_{\Delta \Delta}$ component of the metric tensor decreases with increasing $\Delta$ almost like $1 / \Delta^{2}$, while $\gamma_{\phi \phi}$ is approximately linear in $\Delta$. 


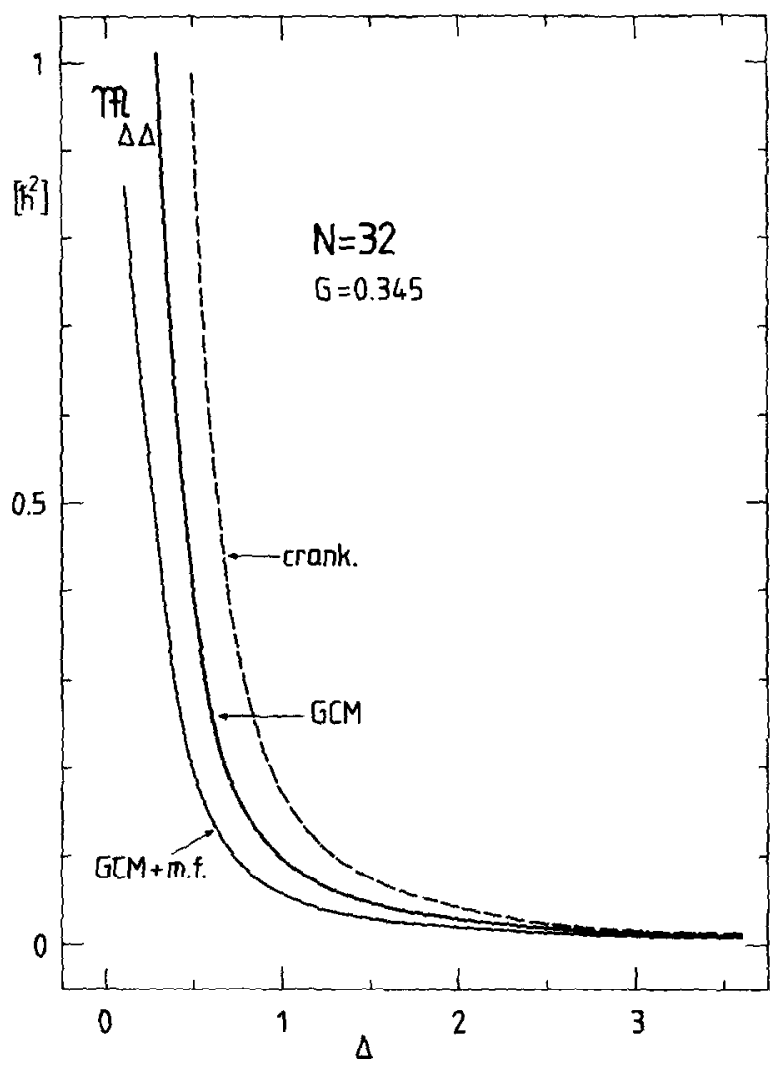

Fig. 2. The same as in fig. 1, but for the mass parameters $\mathscr{M}_{\Delta \Delta}$.

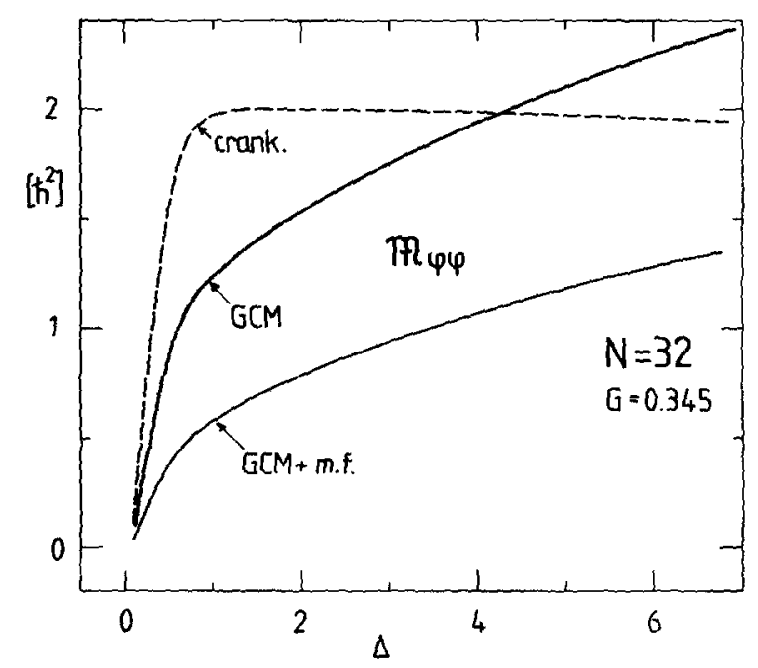

Fig. 3. The same as in fig. 1, but for the mass parameters $\mathscr{M}_{\phi \phi}$. 


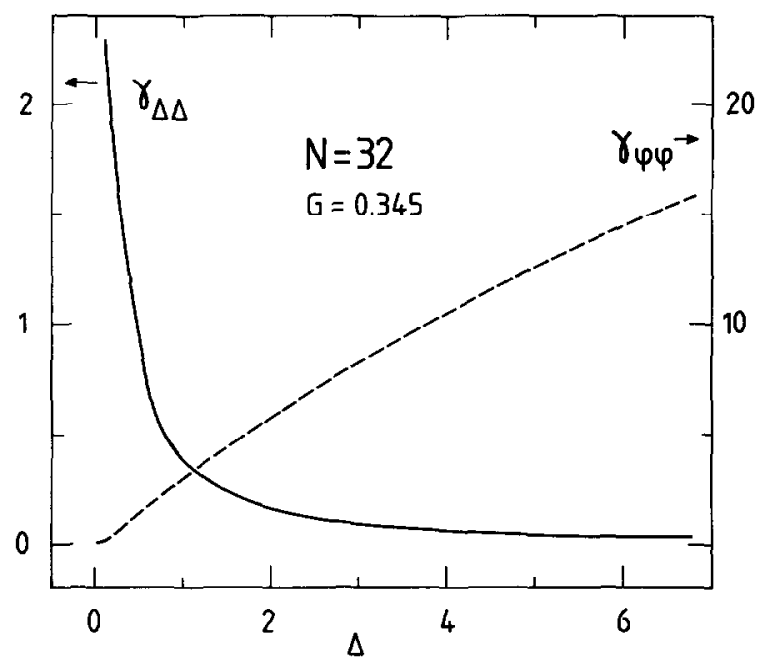

Fig. 4. The widths $\gamma_{\Delta \Delta}$ and $\gamma_{\phi \phi}$ of the overlap of the BCS functions as functions of $\Delta$.

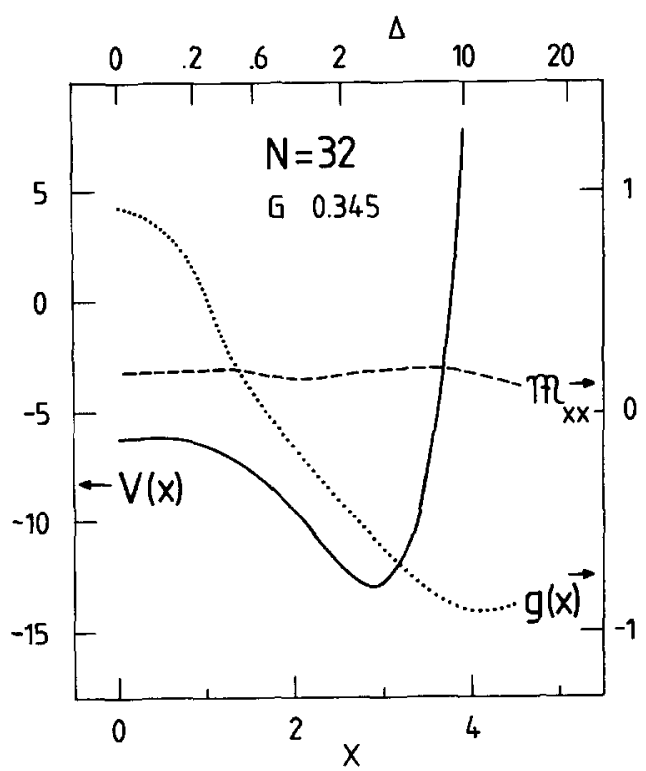

Fig. 5. The collective potential and the mass parameter after the transformation to the new coordinate $x$ (eq. (B.20)). 


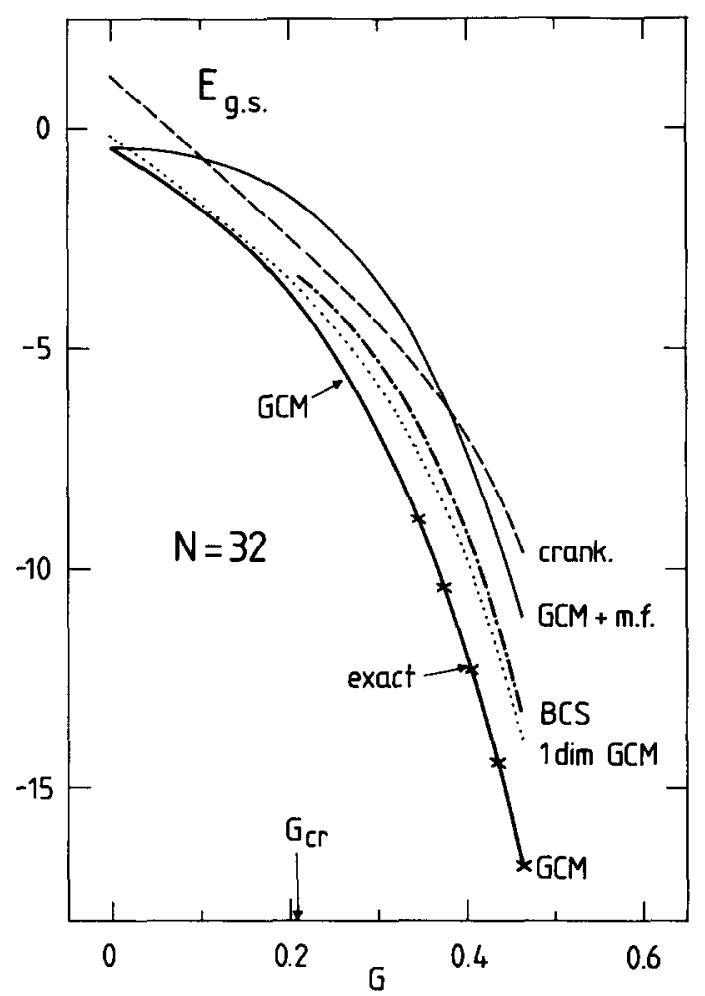

Fig. 6. Dependence of the ground-state energy ( $E_{00}$ in the text) on the pairing strength. The crosses correspond to the exact solutions of the Richardson model.

Having found the potential $V$, the mass and the metric tensors, we can construct numerically the collective hamiltonian (3.1). The method of diagonalization of this hamiltonian is described in appendix B. For simplicity, only the vibrational spectra will be constructed here, but the whole approach can be easily extended to the case of quasirotational states ${ }^{1}$ ). In the first step we performed the transformation (B.10) from $\Delta$ to a new variable $x$ in which the mass parameter $\mathscr{M}_{x x}$ is almost constant. The collective potential and the mass $\mathscr{M}_{x x}$ are plotted in fig. 5 as a function of $x$. Also the function $g(x)$, eq. (B.18), is drawn there. The mass is constant on the average, while $V(x)$ is very similar to the Morse potential. The general solutions of the Morse hamiltonian ${ }^{11}$ ) could be used as a basis for diagonalization of our hamiltonian, but for a basis of harmonic-oscillator eigenstates (in $x$-space) the boundary conditions are simpler.

The ground-state energy for the system of 32 particles is plotted in fig. 6 as a function of the pairing strength $G$. The crosses denote the values of the exact solution by Richardson ${ }^{7}$ ). The results obtained with GCM + GOA for the two collective variables $\Delta, \phi$ (eq. (1.1)) are represented by the thick solid line. They agree 


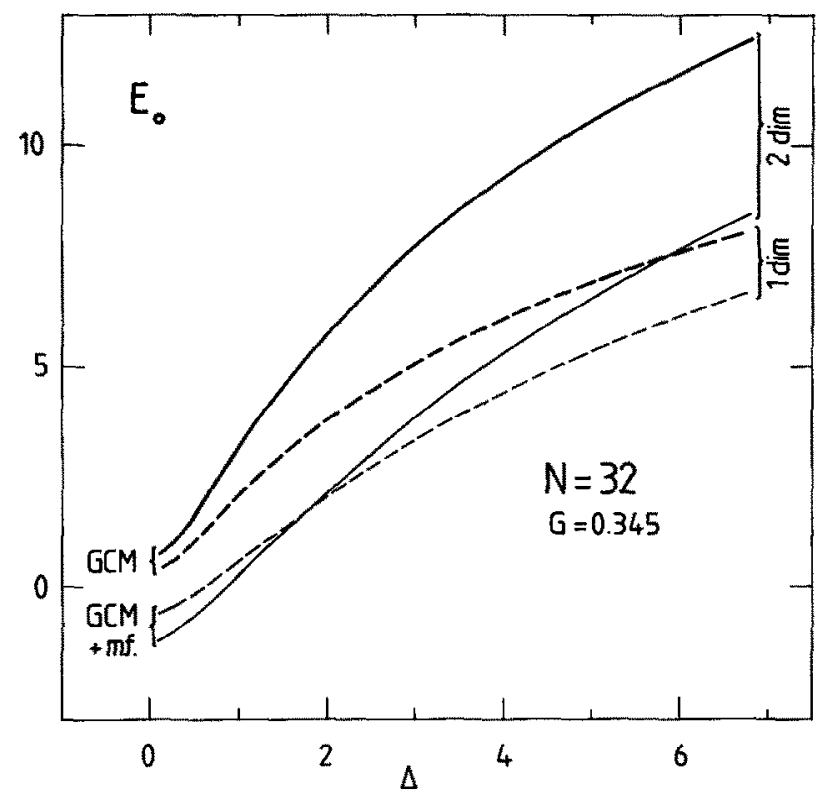

Fig. 7. Zero-point energy versus pairing gap $\Delta$. Solid lines: two-dimensional GCM calculations $(\Delta, \phi)$; dashed lines: one-dimensional calculation $(\Delta)$. Thick lines: results using the full pairing hamiltonian; thin lines: results for the mean-field hamiltonian.

very well with the exact solution. The largest relative error is smaller than $0.8 \%$. The dotted line corresponds to the results obtained within the same approximation but using only one collective coordinate $\Delta$. The agreement with the exact results is much worse but still a bit better than in the case of a stationary BCS calculation (dashed-dotted line in fig. 6). The estimates made with the mean-field hamiltonian (3.11) (thin solid line in fig. 6) and with the collective pairing model (the dashed line) in the cranking approximation ${ }^{1}$ ) are about $40 \%$ too small. The differences between the results for the two- and the one-dimensional models, as well as between the estimates from the full, (3.9), and the mean-field, (3.11), hamiltonians, originate mainly from the different values of the zero-point energies shown in fig. 7.

\subsection{NUMERICAL RESULTS IN A REALISTIC CASE}

GCM calculations performed for more realistic cases than the Richardson model give similar differences between the results obtained with the full pairing hamiltonian and with its mean-field approximation. In fig. 8 both kinds of results are compared for the proton system of ${ }^{240} \mathrm{Pu}$. The deformed $(\varepsilon=0.2)$ Nilsson model single-particle levels were used in this case. The pairing strength and the parameters of the Nilsson potential were standard ${ }^{12}$ ). 


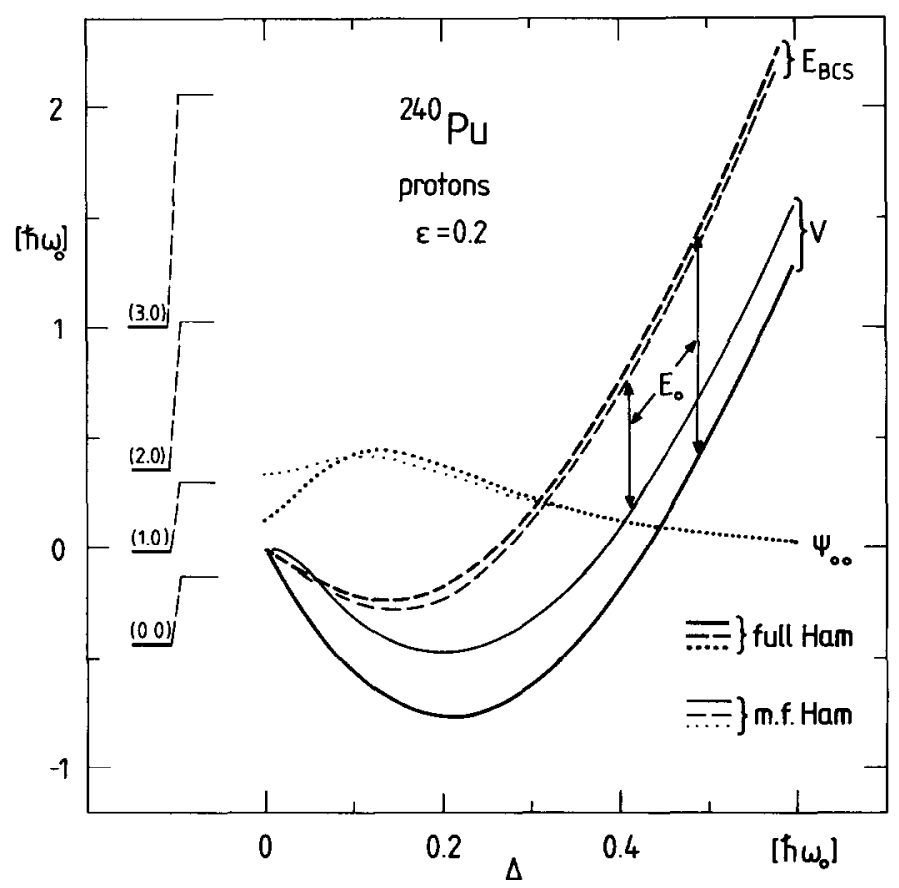

Fig. 8. The collective potentials and the ground-state wavefunctions, normalized as in eq. (3.12), as well as the lowest pairing vibrational states, for the proton system of ${ }^{240} \mathrm{Pu}$ in a realistic Nilsson potential.

The ground-state functions $\Psi_{00}$ are not very different in both cases. Also the lowest vibrational levels obtained with the mean-field pairing hamiltonian are shifted only about $+0.3 \hbar \omega_{0}$ in comparison with the eigenvalues obtained with the full pairing hamiltonian.

The function $\Psi_{00}$ from fig. 8 is already normalized to a volume element 1 :

$$
\int_{0}^{\infty}\left|\Psi_{00}\right|^{2} \mathrm{~d} \Delta=1
$$

The maximum of $\Psi_{00}$ is shifted to smaller values of $\Delta$ in comparison with the BCS equilibrium point or the minimum of the potential $V$. This effect comes from the strong dependence of the mass parameter $\mathscr{M}_{\Delta \Delta}$ on $\Delta$ (see eq. (B.9) or fig. 2).

Of course, our pairing vibrational spectra cannot be directly compared with any experimental data, since the pairing and $\beta$-vibrations are strongly coupled.

The average value of $\mathscr{M}_{\beta \beta^{\prime}}$ weighted with $\left|\Psi_{00}\right|^{2}$ (from fig. 8) is about 3 times larger than that obtained in the BCS equilibrium and reaches approximately the value needed in order to reproduce on the average the lowest $\beta$-vibrational states [see also ref. $\left.{ }^{13}\right)$ ]. This is only a rough estimate; to confirm it quantitatively, one has 
to construct the GCM collective hamiltonian for both pairing and shape vibrations and to diagonalize it. This is a topic for future investigations.

The authors are very grateful to Prof. R. A. Broglia for turning their attention to the pairing vibration problem; also discussions with Dr. S.G. Rohozinski were very helpful. Two of us (A.G. and K.P) are thankful to GSI Darmstadt and Deutsche Forschungsgemeinschaft for grants and to the Universität Regensburg for warm hospitality.

\section{Appendix A}

\section{MICROSCOPIC FORMULAE}

Taking the derivatives of the BCS wave function (1.1), one can easily get the quasiparticle representation of the operators $\partial / \partial \Delta$ and $\partial / \partial \phi$ :

$$
\frac{\partial}{\partial \Delta}|\Delta \phi\rangle=\sum_{\mu} \frac{\tilde{e}_{\mu}}{2 E_{\mu}^{2}} \alpha_{\mu}^{+} \alpha_{-\mu}^{+}|\Delta, \phi\rangle,
$$

where

$$
\begin{gathered}
\tilde{e}_{\mu}=e_{\mu}-\lambda+\Delta \frac{\partial \lambda}{\partial \Delta} \\
\frac{\partial}{\partial \phi}|\Delta \phi\rangle=-i \sum_{\mu} \frac{\Delta}{E_{\mu}} \alpha_{\mu}^{+} \alpha_{-\mu}^{+}|\Delta, \phi\rangle .
\end{gathered}
$$

with the usual definitions of the BCS quantities $u_{\mu}, v_{\mu}, E_{\mu}$ etc. Having formulae (A.1) and (A.2), it is easy to evaluate the microscopic expression for the widths of the overlap

$$
\begin{aligned}
& \gamma_{\Delta \Delta}=\sum_{\nu} \frac{\left(\tilde{e}_{\nu}\right)^{2}}{4 E_{\nu}^{4}}, \\
& \gamma_{\phi \phi}=\sum_{\nu} \frac{\Delta^{2}}{E_{\nu}^{2}} .
\end{aligned}
$$

We now want to construct the collective hamiltonian starting from (i) the single-particle plus pairing hamiltonian

$$
\hat{H}=\sum_{\nu} e_{\nu}\left(c_{\nu}^{+} c_{\nu}+c_{-\nu}^{+} c_{-\nu}\right)-G \sum_{\nu \nu^{\prime}} c_{\nu}^{+} c_{-\nu}^{+} c_{-\nu^{\prime}} c_{\nu^{\prime}}-\lambda(\hat{N}-N),
$$


and (ii) the corresponding mean-field hamiltonian [see also ref. $^{1}$ )]

$$
\begin{aligned}
\hat{H}_{\mathrm{MF}}= & \sum_{\nu} e_{\nu}\left(c_{\nu}^{+} c_{\nu}+c_{-\nu}^{+} c_{-\nu}\right)-G\left(\sum_{\nu} u_{\nu} v_{\nu}\right)\left(\mathrm{e}^{2 i \phi} \sum_{\mu} c_{-\mu} c_{\mu}\right. \\
& \left.+\mathrm{e}^{-2 \imath \phi} \sum_{\mu} c_{\mu}^{+} c_{-\mu}^{+}\right)+G\left(\sum_{\nu} u_{\nu} v_{\nu}\right)^{2}-\lambda(\hat{N}-N) .
\end{aligned}
$$

From both hamiltonians (A.5) and (A.6), we have substracted the term $\lambda(\hat{N}-N)$ describing the fluctuations in the particle number [see also the discussion in ref. $^{3}$ )]. These fluctuations vanish when our projection (integration on the gauge angle $\Phi$ ) is exact, but the term $\lambda(\hat{N}-N)$ helps to write down the further formulae in a more symmetric form.

The matrix elements of $\hat{H}$ appearing in the formulae (3.4)-(3.8) can, after straightforward but tedious calculations, be written in the following form:

$$
\begin{aligned}
& \langle\Delta \phi|\hat{H}| \Delta \phi\rangle=\sum_{\nu} h_{\nu}^{(0)}, \\
& \left\langle\Delta \phi\left|\hat{H} \frac{\vec{\partial}}{\partial \Delta}\right| \Delta \phi\right\rangle=\sum_{\nu} \frac{\tilde{e}_{\nu}}{2 E_{\nu}^{2}} h_{\nu}^{(2)}, \\
& \left\langle\Delta \phi\left|\hat{H} \frac{\vec{\partial}}{\partial \phi}\right| \Delta \phi\right\rangle=-i \sum_{\nu} \frac{\Delta}{E_{\nu}} h_{\nu}^{(2)}, \\
& \left\langle\Delta \phi\left|\frac{\overleftarrow{\partial}}{\partial \Delta} \hat{H} \frac{\vec{\partial}}{\partial \Delta}\right| \Delta \phi\right\rangle_{L}=\sum_{\nu}\left(\frac{\tilde{e}_{\nu}}{2 E_{\nu}^{2}}\right)^{2} 2 h_{\nu}^{(1)} \\
& -G\left\{\sum_{\nu}\left(\frac{\tilde{e}_{v}}{E_{v}^{2}} u_{\nu} \nu_{\nu}\right)^{2}+\left(\sum_{\nu} \frac{\tilde{e}_{v}}{2 E_{v}^{2}} v_{v}^{2}\right)^{2}+\left(\sum_{\nu} \frac{\tilde{e}_{v}}{2 E_{v}^{2}} u_{v}^{2}\right)^{2}\right\}, \\
& \left\langle\Delta \phi\left|\frac{\bar{\partial}}{\partial \phi} \hat{H} \frac{\vec{\partial}}{\partial \phi}\right| \Delta \phi\right\rangle_{L}=\sum_{\nu}\left(\frac{\Delta}{E_{\nu}}\right)^{2} 2 h_{\nu}^{(1)} \\
& -G\left\{\sum_{\nu}\left(\frac{\Delta}{E_{\nu}} 2 u_{v} v_{\nu}\right)^{2}+\left(\sum_{\nu} \frac{\Delta}{E_{v}} v_{v}^{2}\right)^{2}+\left(\sum_{\nu^{\prime}} \frac{\Delta}{E_{\nu}} u_{\nu}^{2}\right)^{2}\right\} .
\end{aligned}
$$

We have used here the notation $h_{v}^{(n)}$ for the coefficients appearing in the quasipar- 
ticle expansion of $\hat{H},($ A.6):

$$
\begin{aligned}
\hat{H}= & \sum_{\nu} h_{\nu}^{(0)}+\sum_{\nu} h_{\nu}^{(1)}\left(\alpha_{\nu}^{+} \alpha_{\nu}+\alpha_{-\nu}^{+} \alpha_{-\nu}\right) \\
& +\sum_{\nu} h_{\nu}^{(2)}\left(\alpha_{\nu}^{+} \alpha_{-\nu}^{+}+\alpha_{-\nu} \alpha_{\nu}\right)+\ldots,
\end{aligned}
$$

where

$$
\begin{aligned}
& h_{\nu}^{(0)}=2 e_{\nu} v_{\nu}^{2}-G\left(\sum_{\mu} u_{\mu} v_{\mu}\right) u_{\nu} v_{\nu}-G v_{\nu}^{4}, \\
& h_{\nu}^{(1)}=\left(e_{\nu}-\lambda\right)\left(u_{\nu}^{2}-v_{\nu}^{2}\right)+2 G\left(\sum_{\mu} u_{\mu} v_{\mu}\right) u_{\nu} v_{\nu}+G v_{\nu}^{4}, \\
& h_{\nu}^{(2)}=2 u_{\nu} v_{\nu}\left(e_{\nu}-\lambda-G v_{\nu}^{2}\right)-G\left(\sum_{\mu} u_{\mu} v_{\mu}\right)\left(u_{\nu}^{2}-v_{\nu}^{2}\right) .
\end{aligned}
$$

The terms underlined in eqs. (A.10)-(A.15) will not appear when we use the mean-field hamiltonian (A.6) instead of the full single-particle plus pairing hamiltonian (A.5). The derivative of (A.8) appearing in (3.4), is equal to

$$
\begin{aligned}
\frac{\partial}{\partial \Delta}\left\langle\Delta \phi\left|\hat{H} \frac{\vec{\partial}}{\partial \Delta}\right| \Delta \phi\right\rangle= & \sum_{\nu} \frac{2\left[\left(e_{\nu}-\lambda\right) \frac{\partial \lambda}{\partial \Delta}-\Delta\right] \tilde{e}_{\nu}+E_{\nu}^{2} \frac{\partial^{2} \lambda}{\partial \Delta^{2}}}{2 E_{\nu}^{4}} h_{\nu}^{(2)} \\
& +\sum_{\nu} \frac{\tilde{e}_{\nu}}{2 E_{\nu}^{2}}\left\{\frac{\left(e_{\nu}-\lambda\right) \tilde{e}_{\nu}}{E_{\nu}^{3}}\left(e_{\nu}-\lambda-G v_{\nu}^{2}\right)-\frac{\Delta}{E_{\nu}}\left(\frac{\partial \lambda}{\partial \Delta}+G \frac{\Delta \tilde{e}_{\nu}}{2 E_{\nu}^{3}}\right)\right. \\
& \left.-G\left(\sum_{\mu} \frac{\left(e_{\mu}-\lambda\right) \tilde{e}_{\mu}}{2 E_{\mu}^{3}}\right) \frac{e_{\nu}-\lambda}{E_{\nu}}+G\left(\sum_{\mu} \frac{\Delta}{2 E_{\mu}}\right) \frac{\Delta \tilde{e}_{\nu}}{E_{\nu}^{3}}\right\} .
\end{aligned}
$$

The terms with $\partial \hat{H} / \partial \Delta$ and $\partial \hat{H} / \partial \phi$ in the expression for the zero-point energy (3.8) appear for $\hat{H}_{\mathrm{MF}}$ only:

$$
\begin{aligned}
\left\langle\Delta \phi\left|\frac{\partial \hat{H}_{\mathrm{MF}}}{\partial \Delta} \frac{\vec{\partial}}{\partial \Delta}\right| \Delta \phi\right\rangle & =-G\left[\sum_{\mu} \frac{\left(e_{\mu}-\lambda\right) \tilde{e}_{\mu}}{2 E_{\mu}^{3}}\right]\left[\sum_{\nu} \frac{\tilde{e}_{\nu}}{2 E_{\nu}^{2}}\left(u_{\nu}^{2}-v_{\nu}^{2}\right)\right], \\
\left\langle\Delta \phi\left|\frac{\partial \hat{H}_{\mathrm{MF}}}{\partial \phi} \frac{\vec{\partial}}{\partial \phi}\right| \Delta \phi\right\rangle & =-2 G\left(\sum_{\mu} u_{\mu} v_{\mu}\right)\left(\sum_{\nu} \frac{\Delta}{E_{\nu}}\right) .
\end{aligned}
$$

The cranking estimates of the mass parameters are ${ }^{1}$ )

$$
\begin{aligned}
& \mathscr{M}_{\Delta \Delta}^{\mathrm{cr}}=\sum_{\nu}\left[\left(e_{\nu}-\lambda\right)^{2}+\left(e_{\nu}-\lambda\right) \Delta \frac{\partial \lambda}{\partial \Delta}+\Delta^{2}\left(\frac{\partial \lambda}{\partial \Delta}\right)^{2}\right] /\left(4 E_{\nu}^{5}\right), \\
& \mathscr{M}_{\phi \phi}^{c r}=\sum_{\nu} \frac{\Delta^{2}}{E_{\nu}^{3}} .
\end{aligned}
$$


The collective cranking hamiltonian has a form very similar to $\left.(3.1)^{1}\right)$ :

$$
\mathscr{H}_{\text {coll }}^{\text {cr }}=\frac{-\hbar^{2}}{2 \sqrt{\operatorname{det} \mathscr{M}^{\text {cr }}}} \frac{\partial}{\partial \Delta} \frac{\sqrt{\operatorname{det} \mathscr{M}^{\text {cr }}}}{\mathscr{M}_{\Delta \Delta}^{\text {cr }}} \frac{\partial}{\partial \Delta}-\frac{\hbar^{2}}{2 \mathscr{M}_{\phi \phi}^{\text {cr }}} \frac{\partial^{2}}{\partial \phi^{2}}+V .
$$

$V$ is here taken as (A.7) without zero-point energy.

\section{Appendix B}

DIAGONALIZATION OF THE COLLECTIVE PAIRING HAMILTONIAN

Because the hamiltonian (3.1) is independent at the gauge angle $\phi$, its eigenfunctions can be written in the form

$$
\Psi_{n m}(\Delta, \phi)=\frac{1}{\sqrt{2 \pi}} \mathrm{e}^{i m \phi} \chi_{n}(\Delta),
$$

where $m=N-N_{0}=0, \pm 2, \pm 4, \ldots$ In addition, for the ground state $m=0$, and in the vibrational band built on top of it, only even solutions are allowed. This implies the following boundary condition for $\chi_{n}(\Delta)$ :

$$
\left(\frac{d \chi_{n}(\Delta)}{d \Delta}\right)_{\Delta=0}=0
$$

In the numerical applications we have considered only this case, i.e. $m=0$.

All the coefficients in the hamiltonian (3.1) are independent of the gauge angle $\phi$, and the collective pairing hamiltonian can be written as

$$
\hat{\mathscr{H}}_{\text {coll }}=-\frac{\hbar^{2}}{2 D} \cdot \frac{\partial}{\partial \Delta} D \mathscr{M}_{\Delta \Delta}^{-1} \frac{\partial}{\partial \Delta}+V_{m}(\Delta)
$$

where $V_{m}$ contains already the rotational energy,

$$
V_{m}=V+Q_{\phi} \hbar m+\frac{\hbar^{2} m^{2}}{2 \mathscr{M}_{\phi \phi}} .
$$

$D$ is given either by

$$
D=\sqrt{\gamma_{\Delta \Delta} \gamma_{\phi \phi}}
$$

in the GCM case, or by

$$
D=\sqrt{\mathscr{M}_{\Delta \Delta}^{\mathrm{cr}} \mathscr{M}_{\phi \phi}^{\mathrm{cr}}}
$$

for the cranking hamiltonian ${ }^{1}$ ).

The hermicity condition for (3.1) (or (B.3)) gives the following normalization of $\chi_{n}$ :

$$
\int_{0}^{\infty} \chi_{n}^{*}(\Delta) \chi_{n^{\prime}}(\Delta) D \mathrm{~d} \Delta=\delta_{n n^{\prime}}
$$


Our task is to find a set of orthogonal functions $\phi_{i}$ with the weight $D$ and to evaluate the expansion of $\chi_{n}$ :

$$
\chi_{n}=\sum_{i=1}^{\infty} c_{n i} \varphi_{i}
$$

i.e. to diagonalize the hamiltonian (B.3) in the basis $\phi_{i}$. Of course, our basis has to be chosen in the optimal way, i.e. the expansion (B.7) has to be rapidly convergent. We verified numerically that the expansion (B.7) in the basis of the harmonic-oscillator states is very slowly convergent. A much better convergence can be reached when one performs a transformation from $\Delta$-space to a coordinate $x$ in which the mass parameter

$$
\mathscr{M}_{x x}=\mathscr{M}_{\Delta \Delta}\left(\frac{\partial \Delta}{\partial x}\right)^{2}
$$

is approximately constant.

The mass parameter $\mathscr{M}_{\Delta \Delta}$ evaluated for the full (or the mean-field) pairing hamiltonian or in the cranking model is a rapidly decreasing function of $\Delta$ which can be approximated by the function

$$
\mathscr{M}_{\Delta \Delta} \simeq b /\left(\Delta+\Delta_{0}\right)^{2} .
$$

After performing the transformation

$$
x=\ln \left(1+\Delta / \Delta_{0}\right)^{\sqrt{b / \mu}},
$$

the mass parameter (B.8) is approximately constant,

$$
\mathscr{M}_{\text {xx }} \simeq \mu \text {. }
$$

So our basic states will be generated by the hamiltonian

$$
\hat{H}_{\mathrm{B}}=-\frac{\hbar^{2}}{2 \mu} \frac{\mathrm{d}^{2}}{\mathrm{~d} x^{2}}+\frac{1}{2} \mu \omega^{2} x^{2} .
$$

Because of the condition (B.2) we pick up the even eigenstates only:

$$
\hat{H}_{\mathrm{B}} \psi_{i}=\left(i+\frac{1}{2}\right) \psi_{i}, \quad i=0,2,4, \ldots
$$

The magnitude of $\mu$ is chosen in order to approximate in the best way the potential $V[\Delta(x)]$, and $\omega$ is determined from the plateau condition $\left(\partial E_{i} / \partial \omega \simeq 0\right)$. The normalization of the eigenstates $\psi_{i}$ is

$$
\int_{0}^{\infty} \psi_{i}^{*} \psi_{j} \mathrm{~d} x=\delta_{i j}
$$

The basis (B.12)-(B.14) is much more convenient from the numerical point of view than the basis of the two-dimensional harmonic oscillator whose eigenfunctions are are orthogonal with weight $x$.

The set of functions

$$
\phi_{i}(\Delta)=\left[D \sqrt{\frac{1}{6} \mu}\left(\Delta+\Delta_{0}\right)\right]^{-1 / 2} \psi_{l}[\Delta(x)]
$$


can be used in order to perform the expansion (B.7), i.e. to diagonalize the hamiltonian (B.3). The matrix element

$$
\mathscr{H}_{i j}=\int_{0}^{\infty} \varphi_{i}^{*}(\Delta) \hat{\mathscr{H}}_{\mathrm{coll}} \varphi_{j}(\Delta) D \mathrm{~d} \Delta
$$

can be transformed after some algebra to an integral in $x$-space:

$$
\begin{aligned}
\mathscr{H}_{i j}= & \frac{1}{2} \int_{0}^{\infty} \frac{\mathrm{d} \psi_{i}^{*}}{\mathrm{~d} x} \frac{\mathrm{d} \psi_{j}}{\mathrm{~d} x} \frac{\mathrm{d} x}{\mathscr{M}_{x x}} \\
& -\frac{1}{4} \int_{0}^{\infty} \frac{\mathrm{d}}{\mathrm{d} x}\left(\psi_{i}^{*} \psi_{j}\right) \frac{\mathrm{d} g(x)}{\mathrm{d} x} \frac{\mathrm{d} x}{\mathscr{M}_{x x}} \\
& +\int_{0}^{\infty} \psi_{i}^{*}\left\{V[\Delta(x)]+\frac{1}{8}\left[\frac{\mathrm{d} g(x)}{\mathrm{d} x}\right]^{2} / \mathscr{M}_{x x}\right\} \psi_{j} \mathrm{~d} x,
\end{aligned}
$$

where

$$
g(x)=\ln \left\{D[\Delta(x)] \sqrt{\frac{1}{6} \mu}\left[\Delta(x)+\Delta_{0}\right]\right\}
$$

and $\mathscr{M}_{x x}(x)$ are rather slowly varying functions of $x$ (see fig. 5). Note that the expression (B.17) is written in such a symmetric form that no derivative of the mass function $\mathscr{M}_{x x}$ is involved, which is very comfortable from a numerical point of view.

\section{References}

1) D.R. Bès, R.A. Broglia, R.P.J. Perazzo and K. Kumar, Nucl. Phys. A143 (1970) 1

2) A. Góźdź, Phys. Lett. 152B (1985) 281

3) A. Góźdź, K. Pomorski, M. Brack and E. Werner, Nucl. Phys. A442 (1985) 26

4) C.D. Siegal and R.A. Sorensen, Nucl. Phys. A184 (1972) 81

5) A. Faessler, F. Grümmer and A. Plastino, Z. Phys. 260 (1973) 305

6) T. Une, A. Ikeda and N. Onishi, Prog. Theor. Phys. 55 (1976) 498

7) A. Góźdź et al., to be published

8) P. Ring and P. Schuck, The nuclear many-body problem, (Springer, New York, 1980)

9) R.W. Richardson, Phys. Rev. 141 (1966) 949

10) H. Hofmann, Z. Phys. 250 (1977) 14

11) S. Flügge, Practical quantum mechanics (Springer, New York, 1974)

12) S.G. Nilsson, C.F. Tsang, A. Sobiczewski, Z. Szymański, S. Wycech, C. Gustafson, I.L. Lamm, P. Möller and B. Nilsson, Nucl. Phys. A131 (1969) 1

13) S.G. Rohozínski, J. Dobaczewski, B. Nerlo-Pomorska, K. Pomorski and J. Srebrny, Nucl. Phys. A292 (1977) 66

14) A. Góźdź, Communications JINR, Dubna, E4-82-561, 1982

15) C. Quesne, J. Math. Phys. 14 (1973) 366 